\title{
BMI open Elevated HIV prevalence and risk behaviours among men who have sex with men (MSM) in Vietnam: a systematic review
}

\author{
Macarena C García, Samantha B Meyer, Paul Ward
}

To cite: García MC, Meyer SB, Ward P. Elevated HIV prevalence and risk behaviours among men who have sex with men (MSM) in Vietnam: a systematic review. BMJ Open 2012;2:e001511. doi:10.1136/bmjopen-2012001511

- Prepublication history and additional material for this paper are available online. To view these files please visit the journal online (http://dx.doi.org/10.1136/ bmjopen-2012-001511)

Received 29 May 2012 Accepted 28 August 2012

This final article is available for use under the terms of the Creative Commons Attribution Non-Commercial 2.0 Licence; see http://bmjopen.bmj.com

Discipline of Public Health, Flinders University, Adelaide, South Australia, Australia

\section{Correspondence to} Macarena C García; mgarcia@usaid.gov

\section{ABSTRACT}

Objectives: To review and analyse original studies on HIV prevalence and risk behaviours among men who have sex with men (MSM) in Vietnam.

Design: Systematic literature review. Comprehensive identification of material was conducted by systematic electronic searches of selected databases. Inclusion criteria included studies conducted from 2002 onwards, following a systematic review concluding in 2001 conducted by Colby, Nghia Huu and

Doussantousse. Data analysis was undertaken through the application of both the Cochrane Collaboration and ePPI Centre approaches to the synthesis of qualitative and quantitative studies.

Setting: Vietnam.

Results: Sixteen studies, undertaken during 2005-2011, were identified that met the inclusion criteria. The analysis showed that HIV prevalence among MSM in Vietnam has increased significantly (eg, from $9.4 \%$ in 2006 to $20 \%$ in 2010 in Hanoi) and that protective behaviours, such as condom use and HIV testing and counselling, continue at inadequately low levels.

Conclusions: Increasing HIV prevalence and the lack of effective protective behaviours such as consistent condom use during anal sex among MSM in Vietnam indicate a potential for a more severe HIV epidemic in the future unless targeted and segmented comprehensive HIV prevention strategies for MSM in Vietnam are designed and programmes implemented.

\section{BACKGROUND}

The HIV/AIDS epidemic in Vietnam is still in a concentrated phase, with the highest prevalence rates found among specific populations at higher risk; these include injecting drug users (IDU), female sex workers (FSW) and men who have sex with men (MSM). As documented by the Ministry of Health Estimates and Projections Project Report, ${ }^{1}$ MSM populations are larger than those of the other groups, and are primarily concentrated in urban areas such as Ho Chi Minh City (64 247), the Red River Delta (60 698), the Mekong River Delta

\section{ARTICLE SUMMARY}

Article focus

- Rapidly rising prevalence rates among men who have sex with men (MSM) in Vietnam draws concern from the region and the world. Multiple epidemiological and behavioural studies have addressed HIV prevalence and risk behaviours among MSM in Vietnam, the first study dating back to 1993.

- The current study will review and analyse original studies on HIV prevalence and risk behaviours among men who have MSM in Vietnam.

Key messages

- Although a systematic review of research on Vietnamese MSM and HIV epidemiology was published in 2004, the results presented only include original studies conducted up to 2001 . Since that time, over a dozen studies on MSM in Vietnam have been conducted and are the topic of this review.

- This work starts off where the previous systematic review left off. It finds that the majority of study findings published and/or presented at national/international conferences reveal rapidly increasing rates of HIV infection and an alarming shift in HIV epidemiology among this high-risk population in Vietnam within the last decade.

- This work highlights the need for large-scaletargeted and MSM-friendly prevention interventions for MSM in Vietnam to address the risks posed by low consistent condom use; low lubrication use; high levels of unprotected anal intercourse; and multiple and concurrent sexual partnerships.

Strengths and limitations of this study

- The current study design employed data extraction and validation techniques. Two academics validated and independently scored the data throughout this systematic literature review. The researchers addressed and resolved conflicts in the data.

- One limitation of the study included the fact that the researchers were not blinded to the purpose of the study.

- Another limitation includes that only studies published in English were included; studies published in other languages were omitted. 
(73 727) and Hanoi (35 436). In these urban centres, IDU and FSW population estimates are lower.

According to UNAIDS, ${ }^{2}$ prevalence in the general population is estimated at $0.53 \%$ and an estimated 243000 Vietnamese were living with HIV and/or AIDS in 2009. Of all reported HIV cases, $78.9 \%$ are in the age group 20-39, with males accounting for $85.2 \%$ of total reported HIV cases. The average age of people living with HIV is decreasing and heterosexual transmission is becoming more significant. $^{2}$

Unlike Thailand to the west, the epidemic in Vietnam is not as severe. UNAIDS $^{2}$ reports that the epidemics in Ho Chi Minh City (HCMC) and the north-east coast initiated earlier, while epidemics in other parts of the country are much more recent. According to UNAIDS, ${ }^{2}$ "this variability has resulted in a geographic concentration of HIV cases in large cities and provinces where the local HIV epidemic in groups of IDUs, FSWs and MSM is substantial."

Male-to-male sexual contact has been an important route of HIV-1 infection since HIV/AIDS was first identified nearly 30 years ago. In the past few years, there has been increased concern about new, newly identified and resurging epidemics of HIV infection in men who have MSM on a global level. ${ }^{3}{ }^{4}$ Against the backdrop of low and declining adult HIV prevalence in most countries, MSM continue to be disproportionately affected by HIV infection. ${ }^{3}$ In Asia, MSM have 18.7 times the odds of being HIV infected compared with someone in the general adult population. ${ }^{3}$ In recent years, scattered epidemiological research has identified high HIV prevalence among MSM in several Asian countries, with varying degrees of study findings and conclusions across countries. Recent data made available through the presentation of preliminary Integrated Biological and Behavioral Survey (IBBS) results suggest an exponential increase in HIV prevalence among MSM in both Hanoi and Ho Chi Minh City, from $9.4 \%$ and $5.3 \%$ in 2006, respectively, to $20 \%$ and $14 \%$ in 2009 , respectively. ${ }^{5}$

Rapidly rising prevalence rates among MSM in Vietnam draws concern from the region and the world. Multiple epidemiological and behavioural studies have addressed HIV prevalence and risk behaviours among MSM in Vietnam, the first study dating back to $1993 .{ }^{6}$ Although a comprehensive and systematic review of research on Vietnamese MSM and risk factors for HIV was undertaken by Colby et $a l^{7}$ the results capture original studies conducted up to 2001. Since that time, over a dozen biological and behavioural studies on MSM in Vietnam have been conducted and are the topic of this review. This systematic review sets forth a summary analysis of identified additional studies, highlighting the current state of HIV prevalence and risk behaviour among this at-risk population.

\section{METHOD}

\section{Search strategy}

Original studies investigating HIV prevalence and risk behaviours among MSM in Vietnam were identified by searching both electronic databases (PubMed, BioMed, MEDLINE and Google Scholar) and conference proceedings. Guided by the search protocol applied in a similar review of global scope in Baral et $a l^{3}{ }^{3}$ the following medical subject heading (MESH) terms were used as title keywords in database searches conducted: HIV AND (MSM OR homosexual AND Viet*) OR (men who have sex with men AND Viet*) OR (Human Immune Deficiency Syndrome) and limited to reports in the English language. Additional studies were also identified through cross-referencing, examination of the bibliographies of retrieved articles and making contact with primary researchers and authors in Vietnam.

Inclusion criteria included the following: studies on HIV prevalence and risk behaviour data among MSM populations in Vietnam (including homosexual, bisexual, male sex workers (MSWs) and transgenders); publication in a peer-reviewed journal; and, an abstract at a conference. Gray literature was identified and included on a case-by-case basis. For example, if the studies were not published in a peer-reviewed journal, though commissioned by the Government of Vietnam and/or an International Non-Governmental Organization, the studies were included.

Exclusion criteria were adapted from similar studies with global breadth, which resulted in a standardised method of excluding studies that did not meet rigorous pre-determined minimum standards. ${ }^{3}$ Articles/abstracts presenting reviews of several studies were omitted, and only original study findings were included in this systematic review. The ePPI Centre quality and relevance appraisal framework, ${ }^{8}$ discussed in the proceeding section, was used to summarise the weight of evidence each study could contribute to the review's findings, and was an important component of the exclusion criteria.

\section{Data extraction and analysis}

The initial search strategy yielded a total of 326 papers. This number was subsequently reduced through a number of stages, using the inclusion and exclusion criteria outlined above. However, only the final 16 studies were assessed against the ePPI Centre quality and relevance appraisal framework. ${ }^{8}$ The titles of the papers were reviewed for geographic and substantive relevance, which reduced the number to 12. Copies of these papers were obtained and respective bibliographies were reviewed in order to identify additional papers of relevance. Primary researchers and authors in Vietnam with published expertise in MSM issues were contacted which resulted in the collection of 33 additional documents not available in the database searches conducted (ie, conference papers, presentations and preliminary study findings). The last step was to apply the inclusion and exclusion criteria to the additional documents retrieved. The final number of papers for review was 16 (figure 1).

Data extraction was performed using a template designed for this purpose. For all studies in this 


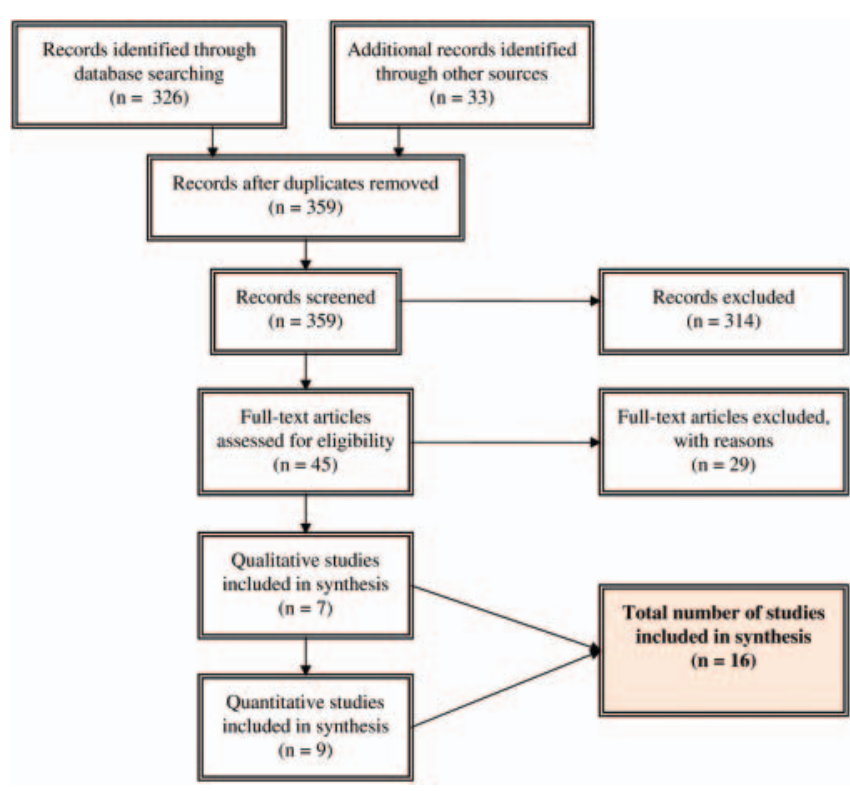

Figure 1 Literature search flow diagram. ${ }^{22}$

review, the following data were extracted from original publications:

1. Descriptive and substantive data: (a) first author and year of publication; (b) study site and period; (c) sampling methods; (d) sampling size and age of participants; (e) methods and results of HIV infection detection; (f) reported risk behaviours and (g) outcome measures.

2. ePPI quality and relevance appraisal: (a) trustworthiness of results judged by the quality of the study within the accepted norms for undertaking the particular type of research design used in the study; (b) appropriateness of the use of the study design for addressing the systematic review's research question; (c) appropriateness of focus for the research for answering the review question and (d) judgement of overall weight of evidence based on the assessments made for each of the criteria above.

Data extraction and validation were carried out by one of authors (MCG) and abstraction methods and data extraction were independently scored and validated by a second academic (SBM). Conflicts between abstractors were settled by subsequent discussion and when appropriate, by contacting the authors of the study in question for further verification. Abstractor and reviewers were not blinded to the purpose of this study, nor blinded to author affiliations. Findings from extracted studies were analysed with a focus on exploring HIV prevalence and risk behaviour among MSM in Vietnam.

\section{RESULTS}

The systematic review of original studies yielded the identification of two main themes. The first theme is formal and includes government-owned data from biological and behavioural surveillance studies carried out in Vietnam in 2006 and 2009. These data are considered official and are often cited in peer-reviewed publications, conference papers/presentations and reports to the United Nations General Assembly Special Session on HIV/AIDS (UNGASS). The second theme is less formal and includes studies carried out by independent researchers, universities and non-governmental organisations. These studies address gaps in existing knowledge about HIV risk and behaviour, and include more comprehensive data sets than those found in official surveillance reports.

\section{Biological and behavioural surveillance}

MSM are not part of the national surveillance system which tracks HIV incidence and prevalence among FSW and IDU, among others. ${ }^{9}$ However, MSM are now a target group for future surveillance efforts in Vietnam, having been included in the Estimates and Projections Project of 2009. ${ }^{1}$ According to Fontaine (Fontaine C. Personal communication) at the United Nations Joint Programme for HIV/AIDS (UNAIDS) office in Vietnam, MSM have been included as a 'pilot' group in the newly established HIV sentinel surveillance plus behavioural surveillance initiative in the 2010 and 2011 rounds (data not yet available). To date, the most prominent biological and behavioural studies to include MSM as an at-risk group for HIV infection have been the two IBBS rounds, $2005-2006^{10}$ and $2009 .^{5}$

The 2009 IBBS sampled 1596 MSM in Hanoi, Hai Phong, Ho Chi Minh City, and Can Tho. Data were not disaggregated between male identified MSM, and transgenders, but was disaggregated by men reporting transactional sex and those not. Along with HIV prevalence and risk behaviours, sexually transmitted infections were also measured (see table 1, below).

Although HIV prevalence was highest among MSM in Hanoi not reporting transactional sex (19.9\%), prevalence in Hai Phong and Ho Chi Minh City is equally concerning, at $16.6 \%$ and $14.4 \%$ among MSM not reporting transactional sex. ${ }^{5}$ The 2009 IBBS data show that MSM reporting transactional sex have a lower HIV prevalence rate in all provinces where IBBS conducted

Table 1 STI* prevalence among MSM (2009 IBBS)

\begin{tabular}{|c|c|c|}
\hline Province & $\begin{array}{l}\text { MSM who reported } \\
\text { transactional } \\
\text { sex }(\%)\end{array}$ & $\begin{array}{l}\text { MSM who did not } \\
\text { report transactional } \\
\text { sex }(\%)\end{array}$ \\
\hline Hanoi & 18.7 & 13.4 \\
\hline Hai Phong & No data available & 7.5 \\
\hline $\begin{array}{l}\text { Ho Chi } \\
\text { Minh City }\end{array}$ & 21.5 & 21.1 \\
\hline Can Tho & 17.7 & 17.3 \\
\hline
\end{tabular}


surveys, except for Can Tho where MSM reporting transactional sex had nearly a twofold HIV prevalence rate compared to MSM who did not report transactional sex (8.9\% and $5 \%$, respectively). The biological and behavioural survey from Ho Chi Minh City reveals a 16\% HIV prevalence rate, ${ }^{11}$ slightly lower than the results from the 2009 IBBS, $^{5}$ which showed a prevalence rate of 16.4\%. Approximately 47\% of Ho Chi Minh City MSM in the Ho Chi Minh City study reported having sex with another male for transactional purposes in the last 12 months, with $16 \%$ reporting to have injected drugs in their lifetime and $41.7 \%$ of those having injected in the last year. ${ }^{5}$ However, the 2009 IBBS shows higher rates of injecting drug use among HIV infected MSM in Hanoi, Ho Chi Minh City and Can Tho.

According to preliminary data from the 2009 IBBS, ${ }^{5}$ HIV prevalence among MSM in Hanoi and Ho Chi Minh City has significantly increased since the first IBBS round in 2006. Among MSM not reporting transactional sex in Hanoi, HIV prevalence nearly doubled (from $11 \%$ to $20 \%$ ), and in Ho Chi Minh City HIV prevalence among MSM not reporting transactional sex nearly tripled going from $6 \%$ in 2006 to $16 \%$ in $2009 .{ }^{5}$

Among both MSM reporting transactional sex and those who did not, a significant percentage reported sex with regular female partners, as well as FSW. In Can Tho, Ho Chi Minh City and Hanoi, more than $45 \%$ of MSM who reported transactional sex also reported having a regular female sexual partner $(56 \%, 47 \%$ and $51 \%$, respectively). Only $25 \%$ of MSM in Can Tho reported having sex with an FSW, 18\% in HCMC and 20\% in Hanoi reported the same. ${ }^{5}$ Conversely, sex with MSWs was much lower than sex with FSWs among MSM reporting transactional sex. ${ }^{5}$ MSM who did not report transactional sex reported higher rates of sex with consensual partner/s and sex with a regular female partner. $^{5}$

Comparisons between the data from 2006 and 2009 rounds of IBBS reveal that consistent condom use with consensual partners among MSM reporting no transactional sex decreased in Ho Chi Minh City, going from $38 \%$ in 2006 to $30 \%$ in 2009. Similarly, among this group of MSM, consistent condom use with regular female partners decreased slightly, from $27 \%$ to $24 \%$. However, dramatic increases are noted in Hanoi where consistent condom use with consensual partners went from $30 \%$ in 2006 to $65 \%$ in 2009 . There was also an increase in reported consistent condom use with regular female partners among MSM in Hanoi, from 23\% in
2006 to $32 \%$ in $2009 .^{5}$ Although the increase in consistent condom use is important and worth noting, reported consistent condom use among MSM remains low and comparable to low condom use among other at-risk groups in Vietnam, such as commercial sex workers-the only outlier being condom use among MSM with consensual partner in Hanoi (see table 2).

\section{Epidemiological and behavioural studies}

In addition to IBBS rounds, many independent researchers, organisations and universities have carried out epidemiological and behavioural studies on MSM in Vietnam. Le and Clatts ${ }^{12}$ conducted a behavioural study in 2005 among 110 MSWs in Hanoi. Also in 2005, Colby, Minh and Toan ${ }^{13}$ carried out a study on HIV risk and prevalence among MSM living in rural Vietnam, the first study of its kind to study this population in a rural setting. Nguyen, Schoenbach, Huynh and $\mathrm{Le}^{14}$ presented behavioural data the 7th Vietnamese Education Foundation Fellows and Scholars Conference, collected from over 6000 MSM through an online forum. Colby and Mimiaga ${ }^{15}$ have also made available preliminary findings from their study of MSW in Ho Chi Minh City, undertaken in 2009 and 2010. Finally, Nguyen ${ }^{11}$ has released preliminary findings from a biological and behavioural survey conducted in Ho Chi Minh City in 2010, among 300 MSM, which was independent from the national 2009 IBBS. The latter two studies represent unpublished data which have not been peer reviewed and therefore are not included in this formal systematic review. However, the preliminary data from these studies are critical to understanding the HIV risk behaviours among MSM in Vietnam, especially among MSWs, a risk group that has been historically understudied.

In the Colby and Mimiaga ${ }^{15}$ study on MSW, $41 \%$ reported having sex with female/s, $8 \%$ reported having sex with FSW and 16\% having sex with MSW in 2009. Those figures decreased in 2010 to $35 \%, 5 \%$ and $9 \%$, respectively. ${ }^{15}$ In comparison, Nguyen et $a l^{14}{ }^{14}$ online study yielded very different results, with only $7.6 \%$ of 3231 MSM surveyed reporting both male and female sexual partners. In the Colby and Mimiaga study, ${ }^{15} 36 \%$ of MSM reported having unprotected anal intercourse in the past month in 2009 , with a decrease to $22 \%$ in 2010 among the same MSM study cohort. Nearly a quarter of MSM in Colby and Mimiaga's study ${ }^{15}$ reported unprotected anal sex with male client/s in 2009 and 2010 (22\% and 21\%, respectively).

Table 2 Consistent condom use: comparison between FSW and MSM (2009 IBBS)

\begin{tabular}{|c|c|c|c|c|}
\hline Province & $\begin{array}{l}\text { Venue-based } \\
\text { FSW (\%) }\end{array}$ & $\begin{array}{l}\text { Street-based } \\
\text { FSW (\%) }\end{array}$ & $\begin{array}{l}\text { MSM w/consensual } \\
\text { partner }(\%)\end{array}$ & $\begin{array}{l}\text { MSM w/regular female } \\
\text { partner (\%) }\end{array}$ \\
\hline Hanoi & 38 & 33 & 65 & 32 \\
\hline Ho Chi Minh City & 32 & 23 & 30 & 24 \\
\hline
\end{tabular}

Source: Nguyen and Tran ${ }^{5}$.

FSW, female sex workers; MSM, men who have sex with men. 
The Hanoi MSW study by Le and Clatts ${ }^{12}$ found that most men who sell sex in Hanoi came from other provinces $(79 \%)$, with most selling sex for economic survival and the majority reporting their exclusive attraction to women $(74 \%)$. The Le and Clatts study ${ }^{12}$ also revealed that $58 \%$ of MSW had used at least one type of illicit drug in the past $(58 \%)$. For those that reported illicit drug use, they most commonly used drugs in the past 90 days, and the most frequent drug used was the injection of heroin $(50 \%)$. In this particular study, higher levels of condom use were reported than in the Colby and Mimiaga study, ${ }^{15}$ with $65 \%$ of MSW reporting condom use during anal sex. ${ }^{11}$ Of the MSW reporting Heroin injection, $42 \%$ reported having insertive anal sex with their most recent sex client, with no condom use in $47 \%$ of cases. ${ }^{12}$ One-third of the $110 \mathrm{MSW}$ interviewed in Hanoi reported having paid for sex in the last 90 days, with $81 \%$ buying sex from FSW and only $19 \%$ buying sex from other MSW. ${ }^{12}$

Colby et $a l^{13}$ studied rural MSM populations in Khanh Hoa province, particularly focusing on HIV risk and prevalence. Of the 216 MSM living in rural areas, $46 \%$ described their sexual orientation as bisexual, $9 \%$ as heterosexual and $45 \%$ as homosexual. ${ }^{13}$ This was the first survey to confirm that MSM not only live in urban settings, but can also be found in rural areas, where the majority of Vietnam's population live, and are relatively easy to identify. ${ }^{13}$ As with urban MSM, this study revealed that vaginal sex was relatively common, with $36 \%$ of rural MSM engaging in vaginal sex with a female partner in the previous 6 months. However, anal sex with casual male partners was more common, with $47 \%$ of rural MSM having engaged in anal intercourse with a casual male partner in the previous 6 months. All MSM in this study tested for HIV were found to be negative. ${ }^{13}$ However, according to Lowe and Thien, ${ }^{16}$ HIV testing among a group of 800 MSM in Khanh Hoa province revealed a prevalence rate of $1.9 \%$. Table 3, below, provides a summary of HIV prevalence and consistent condom use across all studies included in this review.

\section{DISCUSSION}

The results of this systematic review strongly suggest that HIV prevalence among MSM in Vietnam has been on the rise over time. In fact, the Ho Chi Minh City AIDS Committee $^{17}$ estimates that by 2012 , the number of new HIV infections contracted by MSM in Ho Chi Minh city each year is projected to be higher than the annual number of new infections in each of the other two identified high-risk groups in the city, IDU and FSW. Behavioural data, such as HIV testing, number of sexual partners and unprotected anal intercourse, also suggest reason for concern.

Knowing one's HIV status and associated counselling have been found to be associated with decreased high-risk sexual practices. ${ }^{18}$ Although HIV counselling and testing is increasing among IDU and FSW, it remains inadequately low among MSM, and has not increased since the 2006 round of IBBS which may suggest the need for alternative testing strategies for this at-risk group. ${ }^{5}$ Low rates of HIV testing over time may be a contributing cause to the alarming rise in HIV prevalence among MSM in Vietnam. While male-to-male sex is preferred by most of those surveyed in the studies identified above, significant numbers of MSM are also having female sexual partners due to continued family and societal pressure to conform to masculine norms. The low level of consistent condom use with these women, coupled with already relatively high rates of HIV prevalence among MSM and low rates of protective behaviour with other male sex partners reveals yet another contributing factor to elevated HIV prevalence among MSM in Vietnam. ${ }^{16}$

Nguyen et $a l^{14}$ found that unprotected sex among surveyed MSM correlated with low perception about risk of HIV transmission, HIV prevalence and the number of casual sex partners. The findings of this review identify a need for greater HIV awareness among this group, as well as programmes delivering consistent and segmented prevention messages. Coupled with HIV awareness raising and prevention messages, the data analysed in this review point to the need for greater access to MSM-friendly HIV services, such as HIV testing and counselling, condom/lubrication provision, HIV care and treatment, which would contribute to slowing the pace of HIV infections among this high risk group. For example, given that HIV diagnosis often leads to safer sexual practices, according to the findings of an original study by Nguyen and Kiethly ${ }^{19}$ among People Living with HIV (PHIV) in Vietnam, testing and counselling services need to be expanded and segmented based on the needs of each high-risk group in order to increase HIV testing uptake.

Findings from this review also suggest the need to identify and appropriately address the socio-cultural and economic aspects that influence HIV infection among MSM. $^{20}$ Currently, MSM continue to report not being treated equally when they present themselves to public service providers, such as health clinics, schools or public administration offices. Stigma continues to be a significant barrier to accessing basic and necessary services. ${ }^{21}$ Awareness raising campaigns should also be segmented for greater effectiveness, with the delivery of directed information and messaging to families, government entities and the Vietnamese population.

A limitation of the study design includes the omission of literature published in languages other than English; however, efforts were made to contact MSM experts and practitioners in Vietnam to ensure review's inclusiveness and breadth. Another limitation of the study design is that it included abstraction of both behavioural and biological data sets, and therefore the application of PRISMA had to be combined with the ePPI Quality and Relevance appraisal framework in order to ensure appropriate scoring methodology for diverse studies. 
Table 3 HIV prevalence and consistent condom use among MSM in Vietnam

\begin{tabular}{|c|c|c|c|c|c|}
\hline Location & Year & Population & HIV prevalence & Consistent condom use & Reference \\
\hline \multirow[t]{3}{*}{ National } & 2010 & MSM & No data & $72 \%$ (average) & 14 \\
\hline & 2009 & MSM & $5 \%$ & No data & Fridae MSM Sex Survey \\
\hline & 2009 & MSM & $2 \%$ & No Data & 1 \\
\hline \multirow[t]{13}{*}{ Hanoi } & 2009 & MSM/MSW & MSW: $14.3 \%$ & With & 5 \\
\hline & & & MSM: $19.9 \%$ & $\begin{array}{l}\text { Consensual partners: } 65 \% \\
\text { Regular female partners: } 32 \%\end{array}$ & \\
\hline & 2009 & MSM & $3.8 \%$ & No data & 1 \\
\hline & 2009 & MSW & $3 \%$ & During anal sex acts: $65 \%$ & 12 \\
\hline & 2007 & MSM/MSW & MSW: $29.1 \%$ & MSW data only: & 20 \\
\hline & & & MSM: $37.1 \%$ & $\begin{array}{l}\text { Receptive anal sex: } 28.6 \% \\
\text { Insertive anal sex: } 52.6 \%\end{array}$ & \\
\hline & 2006 & MSM & $9.4 \%$ & With: & 10 \\
\hline & & & & Male consensual partners: $29 \%$ & \\
\hline & & & & Male clients: $33 \%$ & \\
\hline & & & & MSW: $24 \%$ & \\
\hline & & & & Female partners: $24 \%$ & \\
\hline & & & & Female clients: $19 \%$ & \\
\hline & & & & $\mathrm{FSW}^{\star \star \star}: 41 \%$ & \\
\hline Hai Phong & 2009 & MSM/MSW & MSW: $14.8 \%$ & No data & 5 \\
\hline & & & MSM: $16.6 \%$ & & \\
\hline \multirow[t]{21}{*}{ Ho Chi Minh City } & 2011 & MSW & $6.3 \%$ & No data & 15 \\
\hline & 2010 & MSM & $16 \%$ & During anal sex: & 11 \\
\hline & & & & Always: $29.7 \%$ & \\
\hline & & & & Almost always: $37.0 \%$ & \\
\hline & 2009 & MSM/MSW & MSW: $16.4 \%$ & With: & 5 \\
\hline & & & MSM: $14.4 \%$ & $\begin{array}{l}\text { Consensual partners: } 30 \% \\
\text { Regular female partners: } 24 \%\end{array}$ & \\
\hline & 2009 & MSM & $9.4 \%$ & No data & 1 \\
\hline & 2008 & MSM & Total: $8 \%$ & With: & Nguyen TA, Nguyen HT, \\
\hline & & & Transgender: $6.8 \%$ & Casual partners: $50.9 \%$ & Le, GT and Detels R. 2008 \\
\hline & & & Non-transgender: $7 \%$ & Regular partners: $34.2 \%$ & \\
\hline & & & Bisexual: $13.5 \%$ & Male sex workers: $57.9 \%$ & \\
\hline & & & Sex worker: $33.3 \%$ & Foreign partners: $58.1 \%$ & \\
\hline & 2006 & MSM & $5.3 \%$ & With: & 10 \\
\hline & & & & Male consensual partners: $37 \%$ & \\
\hline & & & & Male clients: $51 \%$ & \\
\hline & & & & MSW: $32 \%$ & \\
\hline & & & & Female partners: $17 \%$ & \\
\hline & & & & Female clients: $40 \%$ & \\
\hline & & & & FSW: $47 \%$ & \\
\hline & 2005 & MSM & $5.8 \%$ & $40 \%$ & Family Health \\
\hline & & & & & International, 2005 \\
\hline \multirow[t]{2}{*}{ Can Tho } & 2009 & MSM/MSW & MSW: $8.9 \%$ & No data & 5 \\
\hline & & & MSM: $5.0 \%$ & & \\
\hline \multirow[t]{2}{*}{ Khanh Hoa } & 2008 & MSM & $0 \%$ & Urban: $68 \%$ & 13 \\
\hline & & & & Rural: $58 \%$ & \\
\hline
\end{tabular}

A key strength of this review is its inclusion of behavioural data sets, as these provide insight on the rapid rise of HIV prevalence among MSM in Vietnam.

\section{CONCLUSION}

Rapidly rising prevalence rates among men who have sex with men (MSM) in Vietnam draws concern from the region and the world. Multiple epidemiological and behavioural studies have addressed HIV prevalence and risk behaviours among MSM in Vietnam, the first study dating back to 1993. Although a systematic review of research on Vietnamese MSM and HIV epidemiology was published in 2004, the results presented only include original studies conducted up to 2001. Since that time, over a dozen studies on MSM in Vietnam have been conducted and are the topic of this review. 
This work starts off where the previous systematic review left off. It finds that the majority of study results published and/or presented at national/international conferences reveal rapidly increasing rates of HIV infection and an alarming shift in HIV epidemiology among this high-risk population in Vietnam within the last decade.

This work highlights the need for large-scale-targeted and MSM-friendly prevention interventions for MSM in Vietnam to address the risks posed by:

- Low levels of consistent condom use.

- Low lubrication use.

- High levels of unprotected anal intercourse.

- Multiple and concurrent sexual partnerships.

Acknowledgements Accurate data can be challenging to generate and disseminate in Vietnam. However, the UNAIDS/Nietnam country office and the PEPFAR/Vietnam team have diligently worked with the Government of Vietnam to improve surveillance, MARP mapping and estimates and projections. I would like to thank Christopher Fontaine at UNAIDS/Nietnam and Dr Nguyen Cuong Quoc at FHI/Vietnam for their valuable expertise and timely provision of difficult-to-access data and documentation on MSM in Vietnam.

Contributors MCG designed data extraction tools, extracted data, analysed and scored data, and drafted and revised the paper. She is guarantor. SBM scored and validated the extracted data, contributed to article revisions and provided final approval of the version to be published. PW provided study design, contributed to article revisions and provided final approval of the version to be published.

Competing interests None.

Funding This study was funded in part by Flinders University postgraduate grants and independent funding.

Provenance and peer review Not commissioned; externally peer reviewed.

Data sharing statement Data deposited in the Dryad repository: http://dx.doi. org/10.5061/dryad.85qg3.

\section{REFERENCES}

1. Vietnam Administration for AIDS Control and Prevention. Vietnam HIV/AIDS Estimates and Projections, 2007-2012. Hanoi, Vietnam: Vietnam Ministry of Health, 2009.

2. United Nations Joint Programme for HIV/AIDS. Vietnam-Facts and Figures. UNAIDS/Vietnam 2008. unaids.org.vn (accessed 7 Oct 2009).

3. Baral S, Sifakis F, Cleghorn F, et al. Elevated risk for HIV infection among men who have sex with men in low- and middle-income countries 2000-2006: a systematic review. PLoS Med 2007;4:e339.

4. van Griensven F, van Wijngaardenc JW, Baral S, et al. The global epidemic of HIV infection among men who have sex with men. Curr Opin HIV AIDS 2009;4:300-7.
5. Nguyen AT, Tran VH. HIV/STI integrated biological and behavioral surveillance in Vietnam (IBBS), 2009 (round 2). 4th National Scientific Conference on HIV/AIDS; 1-2 December 2009. Hanoi, Vietnam: National Institute of Hygiene and Epidemiology (NIHE) and Vietnam Administration for HIVIAIDS Control (VAAC), Ministry of Health; 2010.

6. Franklin B. The risk of AIDS in Vietnam: an Audience Analysis of Urban Men and Sex Workers, with Guidelines for Prevention. Hanoi, Vietnam: CARE International in Vietnam, 1993.

7. Colby $\mathrm{D}, \mathrm{Cao} \mathrm{NH}$, Doussantousse $\mathrm{S}$. Men who have sex with men and HIV in Vietnam: a review. (Special issue: HIV prevention for Asian and Pacific Islander men who have sex with men: Identifying needs for the Asia Pacific Region). AIDS Educ Prev 2004;16:45-54.

8. ePPI Centre. Quality and relevance appraisal framework for systematic reviews. London, 2011. http://eppi.ioe.ac.uk/ (accessed 4 Dec 2011).

9. Socialist Republic of Vietnam UNGASS country progress report. Hanoi, Vietnam: United Nations, 2010.

10. Vietnam Ministry of Health. Results from the HIV/STI Integrated Biological and Behavioral Surveillance (IBBS) in Vietnam, 2005-2006. Hanoi, Vietnam: Ministry of Health, 2006.

11. Nguyen CQ. HIV epidemic among men who have sex with men in Ho Chi Minh City. (Unpublished).

12. Le MG, Clatts M. Men selling sex to other men in Hanoi: findings from an ethno-epidemiological study. Hanoi, Vietnam: UNAIDS, 2009. http://www.unaids.org.vn (accessed 13 Dec 2011).

13. Colby D, Minh TT, Toan TT. Down on the farm: homosexual behaviour, HIV risk and HIV prevalence in rural communities in Khanh Hoa province, Vietnam. Sex Transm Infect 2008;84:439-43.

14. Nguyen $\mathrm{Q}$, Schoenbach VJ, Le B, et al. HIV risk behaviors of Vietnamese men who have sex with men: results of a national online survey. The 7th Vietnamese Education Foundation Fellows and Scholars Conference; 3-5 January 2010; New York: Rensselaer Polytechnic Institute, 2010.

15. Colby D, Mimiaga M. Results of research on male sex workers in Ho Chih Minh City. (Unpublished).

16. Lowe D, Thien P. Rapid situation and response assessment of HIV and AIDS programs for men who have sex with men in Vietnam. DRAFT REPORT. Hanoi, Vietnam: Vietnam Ministry of HealthVietnam Administration for AIDS Control and Prevention (VAAC), 2010.

17. Ho Chi Minh City Provincial AIDS Committee. The HIV epidemic in Ho Chi Minh City: where is it going? Ho Chi Minh City, Vietnam: USAID, 2006

18. Holtgrave D, McGuire J. Impact of counselling in voluntary counselling and testing programs for persons at risk for or living with HIV infection. Clin Infec Dis 2007;44:360-3.

19. Nguyen NT, Keithly SC. A qualitative study on the sexual behaviour of people living with HIV in Vietnam. AIDS Care Published Online First: 24 January 2012;24:921-8. doi:10.1080/09540121.2011. 644230

20. Clatts MC, Giang LM, Goldsamt LA, et al. Male sex work and HIV risk among young heroin users in Hanoi, Vietnam. Sex Health 2007;4:261-7.

21. Tran TN, Le TMP, Nguyen TV. Research report: MSM in Vietnamsocial stigma and consequences. Hanoi, Vietnam: STDs/HIV/AIDS Prevention Center (SHAPC), 2009.

22. Liberati A, Altman DG, Tetzlaff $\mathrm{J}$, et al. The PRISMA statement for reporting systematic reviews and meta-analyses of studies that evaluate health care interventions: explanation and elaboration. PLoS Med 2009;6:1-28. 